Revue d'histoire de l'Amérique française

PEVUE D.HISTOIRE DE L'AMÉRIQUE FRANÇAISE

\title{
Québec et la guerre franco-allemande de 1870
}

\section{Alfred Rambaud}

Volume 6, numéro 3, décembre 1952

URI : https://id.erudit.org/iderudit/301533ar

DOI : https://doi.org/10.7202/301533ar

Aller au sommaire du numéro

Éditeur(s)

Institut d'histoire de l'Amérique française

ISSN

0035-2357 (imprimé)

1492-1383 (numérique)

Découvrir la revue

Citer cet article

Rambaud, A. (1952). Québec et la guerre franco-allemande de 1870. Revue

d'histoire de l'Amérique française, 6(3), 313-330.

https://doi.org/10.7202/301533ar d'utilisation que vous pouvez consulter en ligne.

https://apropos.erudit.org/fr/usagers/politique-dutilisation/ 


\title{
QUÉBEC ETT LA GUERRE FRANCO-ALLEMANDE DE 1870
}

\author{
$-\mathrm{I}-$
}

Le 16 juillet 1870 Napoléon III déclarait la guerre à la Prusse. teurs ${ }^{1}$.

Le 18 juillet les journaux de Québec l'annonçaient à leurs lec-

L'Événement, organe de tendances libérales, accompagnait la nouvelle de commentaires chaleureux. Malgré les apparences la France n'est pas le pays agresseur. "Elle n'a fait que prevenir l'ennemi et aller au-devant des coups que celui-ci lui destinait... Son arrogance et ses prétentions se bornent à ceci, qu'elle n'a pas voulu permettre qu'un prince prussien montât sur le trône d'Espagne'."

La victoire de la France est d'ailleurs nécessaire. "Elle est l'organe le meilleur et le plus parfait de la civilisation... Sans la France il ferait nuit en Europe"3. Qui oserait comparer la Prusse et la France? "Ligne de démarcation formidable: ici la vivacité, la gaieté, l'expansion native de la race celtique; là la raideur, la discipline et l'orgueil incommensurable de la patrie allemande... C'est Heine qui disait "La Prusse ne connaît qu'un verbe, qu'elle conjugua à tous les temps, prendre4."

D'ailleurs la victoire française ne saurait faire aucun doute. Tous les facteurs jouent en faveur de Napoléon III: supériorité numérique de son armée ${ }^{5}$; excellence du chassepot; meilleur en-

1. Ma documentation repose presque exclusivement sur le dépouillement méthodique des quatre principaux journaux canadiens-français de Québec en 1870: L'Evénement; le Journal de Québec (tous deux quotidiens); le Courrier du Canada et le Canadien (tous deux hebdomadaires).

2. Article du 21 juillet 1870.

3. 3 août 1870 .

4. 3 août 1870 .

5. 16 juillet 1871 . 
traînement des troupes ${ }^{6}$; unanimité française contre la Prusse où déjà éclatent des troubles; intervention probable de la Russie, de l'Autriche, du Danemark en faveur de la France ${ }^{8}$. Dans son numéro du 28 juillet, 12 jours seulement après le début des hostilités, l'Evénement se risque à une prophétie: "L'Empereur a laissé Paris pour joindre la flotte comme le plus sûr et le plus court chemin pour arriver à Berlin, où il est attendu le 15 Août, jour de sa fête."

Victorieuse la France s'installera définitivement sur le Rhin: conquête légitime?.

Le Courrer du Canada n'est pas moins chaleureux pour la France, mais sa sympathie s'appuie sur des raisons exclusivement religieuses. Il rappelle dans son numéro du 29 juillet qu'il a pris parti contre la France dans la désastreuse campagne d'Italie. "Il était convaincu, et les événements lui ont donné raison, qu'elle faisait fausse route et qu'elle ne travaillait qu'au bénéfice de la Révolution". Mais aujourd'hui la France représente la cause de Dieu. "Dans cette question, comme dans toutes les autres, nous mettons au-dessus de toutes les considérations la sécurité de l'Église, et c'est parce que la France représente en cette occurrence l'idée catholique que nous faisons des vœux pour le succès de ses armes". Déjà le 18 juillet le Courrier du Canada avait affirmé: "La France, malgré 'ses fautes, est encore la protectrice de l'Église et son drapeau couvre à Rome le chef de l'Église. La Prusse, elle, représente le protestantisme et le despotisme, et si Dieu, dans sa colère, donnait la victoire à ses armes, que deviendrait Rome et que deviendrait l'Europe ?"'

Le Journal de Québec et le Canadien adoptent des attitudes plus tièdes. Sans doute tous deux protestent de leur sympathie pour la France, mais la guerre franco-prussienne leur paraît bien futile dans ses causes et en tout cas sans aucun intérêt pour le Canada. "Quelles que soient les causes qui provoquent cette guerre, déclare le Journal

6. 23 juillet. "Les Français ont été soldats et ont fait le service dernièrement en Afrique; les Prussiens n'ont que le titre de soldats. La France met une armée en campagne, la Prusse n'a à lui opposer qu'un peuple armé."

7. 23 juillet. "Il y a eu une émeute a Cologne par suite de la cherté des vivres. On a fait en Bavière des démonstrations populaires contre la guerre."

8. 20 et 23 juillet.

9. 28 juillet. 
de Québec dans son numéro du 16 juillet, elles nous paraissent bien futiles à nous qui sommes placés à une grande distance du théâtre des opérations, à nous qui ...n'avons nul enjeu. Il nous est impossible de penser que ces raisons soient assez graves pour obliger deux armées à s'égorger."10

Le Canadien est encore plus net: "Quant à nous Canadiens, de toutes les origines, de toutes les opinions, pourquoi irions-nous nous échauffer en querelles inutiles ?... Nos intérêts politiques et nationaux ne sont plus du ressort de la politique européenne. L'Amérique est à nous. Laissons la vieille Europe se déchirer en lambeaux. Contemplons avec chagrin ses luttes; étudions, à notre avantage, le grand drame sanglant et destructeur qui se joue par delà l'océan, mais, de grâce, soyons unis et prudents et restons unis pour la paix générale."

Ces appels à la modération n'étaient pas inutiles. La nouvelle de la guerre franco-prussienne avait en effet provoqué dans Québec une vive effervescence ${ }^{11}$. Les Canadiens anglais de la ville avaient pris parti pour la Prusse. Par contre les Irlandais et les Canadiens français souhaitaient avec impétuosité la victoire française ${ }^{12}$.

A cet égard un texte de L'Événement est particulièrement émouvant pour un Français de France. C'est l'Editorial du 29 juillet 1870. Je le reproduis presque en entier.

Il ne manquait pas de sceptiques parmi nous qui croyaient que le sentiment français s'était affaibli et que, par l'effet ordinaire d'une longue séparation, notre amour pour la France s'était lentement effacé de notre cœur.

Ils se sont trompés. "Notre population assiste avee un intérêt passionné au drame qui s'ouvre en Europe, elle té-

10. 16 juillet 1870 .

11. Il faut se rappeler qu'à cette date Québec n'était pas aussi exclusivement canadienne-française qu'aujourd'hui.

12. Cf. à ce sujet le Courrier du Canada du 20 et du 29 juillet. L'Opinion publique, le seul hebdomadaire illustré de Montréal, donne la même note dans un article du 11 août 1871. Dans le même numéro est racontée l'anecdote suivante. "Deux enfants de la Belle France causaient combat dans un caboulot de la Basse-Ville. L'on entonnait la Marseillaise quand entrèrent quatre Allemands. On commence par s'injurier, se provoquer, bref une lutte s'engage... Les Français allaient avoir le dessous et perdre les frontières naturelles de l'auberge, quand leur vint un renfort inespéré dans la personne d'un Irlandais qui criait: "Erin go bragh et Vive la France", et fondit sur les amis de la choucroute. En un clin d'œil tout fut changé: les Prussiens reçurent maintes atteintes dans leur "Rhin" allemand et la victoire resta aux Français". 
moigne de l'admiration aux futurs maîtres du Rhin, elle attend avec confiance la première victoire de Napoléon.

Dans le peuple, dans la jeunesse, même chez les hommes d'affaires d'ordinaire inaccessibles aux émotions, il n'y a qu'un sentiment, une voix, un cri "Vive la France.

...Le sang français se remet à couler dans nos veines comme si rien ne l'avait glacé; et nous acclamons le drapeau de notre ancienne mère-patrie comme s'il n'avait cessé de flotter sur nos têtes...

La France reste pour nous la France. C'est notre seal amour national, la source même de notre patriotisme. Nous estimons l'Angleterre, nous lui sommes reconnaissants de nous avoir donné le plus précieux des biens, la liberté; nous admirons les États-Unis, dont la prospérité nous éblouit; mais nous n'aimons avec passion que la France.

Un peuple ne vaut que par le culte qu'il conserve pour ses traditions... ingrats pour la France maintenant, nous le serions plus tard pour l'Angleterre.

$$
\text { - II - }
$$

Les Québécois attendaient avec confiance les premières victoires de Napoléon III. En fait les désastres succèdent aux désastres: Wissembourg; Froeschwiller; Forbach; Rezonville; Gravelotte; Saint-Privat. Devant ces défaites la presse québécoise réagit avec unanimité dans le même sens: parfois elle nie la défaite; le plus souvent elle en minimise la portée; toujours elle annonce de prochains succès qui ne sauraient tarder.

La tactique est particulièrement nette à l'Événement, décidément le plus francophile des journaux de Québec ${ }^{13}$.

Le 5 àût il annonce: "La division du général Douay... a éprouvé un échec momentané à Wiscembourg. Mais les dépêches prussiennes elles-mêmes admettent que ce léger succès a coûté des pertes terribles à l'armée prussienne... Il est inévitable que dans une guerre moderne il y ait des échecs partiels." Le 11 août, malgré les défaites de Froeschwiller et de Forbach, il claironne: "Si donc les dernières nouvelles se confirment, nous croyons que les Prussiens ont vu leurs plus beaux jours". Le 15 août le maréchal Bazaine, le nouveau commandant en chef des troupes françaises, reçoit un hommage délirant.

13. Son directeur était Hector Fabre, qui sera Commissaire général du Canada à Paris de 1882 à 1910. 
"C'est un espoir qui songe, une pensée qui ne se livre pas, une bonté modeste et réelle, un grand amour du soldat. Une froide et grande énergie". Le 20 août l'Événement transforme la défaite de SaintPrivat en éclatante victoire. De gros titres annoncent:

Brillante victoire des Français

La bataille de la Moselle

Pertes énormes des Prussiens

Des milliers de Français s'enrôlent dans la Mobile

Corps de 20,000 cavaliers arabes pour la France

Le 29 août l'Éditorial proteste contre les affirmations de la presse londonienne qui affirme des défaites françaises. "Les dépêches du Times sont mensongères. Comme plusieurs de nos journaux canadiens-anglais, le Times écrit... pour ses lecteurs."

Le 24 août l'optimisme est encore plus grand. "Les Prussiens sont énormément affaiblis... Maintenant toutes les recrues qu'ils peuvent tirer d'Allemagne sont des paysans ou des ouvriers, dont la plupart sont incapables de soutenir les fatigues de la campagne et qui ne connaissent que le maniement de la fourche et du marteau... Les pertes énormes qu'ils ont éprouvées ont démoralisé toute l'Allemagne."

Le 29 août l'Événement attaque un journal canadien-anglais de Québec qui prétendait que les Français n'avaient jamais battu les Prussiens. "Nous prions bien le rédacteur de cette feuille de savoir un peu son histoire... Depuis près d'un siècle la France a toujours battu la Prusse." Et de citer Valmy, Iéna, Averstaedt, Lutzen, Bautzen, Dresde et beaucoup d'autres. L'article ajoutait: "ce que les Français ont fait, ils le feront encore... Les premières batailles n'ont que l'importance d'affaires d'avant-postes... Les événements passés et actuels démontrent incontestablement la supériorité de l'élément celtique sur l'élément saxon."

Les autres journaux laissent percer plus de clairvoyance, mais n'abandonnent pas un instant l'espoir d'un triomphe de la France. Le Journal de Québec vante l'admirable retraite du Maréchal Bazaine: "que le roi Guillaume s'avance aujourd'hui sur Paris, il saura ce que coûte en temps de guerre un voyage à la capitale de la France $^{14}$ ". Le Canadien suggère que "les défaites françaises sont trop

14. 30 août. 
mystérieuses pour qu'elles ne couvrent pas une tactique"15. Il reste une raison d'espérer. "La France est riche. Le nerf de la guerre, l'argent, ne lui fait pas défaut. Elle peut donc prolonger sans gêne la guerre pendant plusieurs années. Au contraire la Prusse est sans crédit. Elle ne peut longtemps soutenir la lutte sans s'épuiser. La Prusse a manqué son coup ${ }^{16 "}$ ". Le Courrier du Canada prévoit dans son numéro du 23 aoât "qu'un second Iéna s'ajoutera aux glorieuses annales militaires de la France". Le 2 septembre il publiait in extenso un article de Louis Veuillot, "La France et la Prusse". "La Prusse est le péché de l'Europe; née du protestantisme, son premier établissement lui fut fait par l'apostasie: elle a grandi dans le délire et par la complicité de l'impiété philosophique. Après Albert de Brandebourg, l'apostat, son second fondateur est Frédéric l'athée."

Les Québécois eux aussi suivaient avec anxiété les nouvelles de la guerre. C'était la matière de toutes les conversations, au coin des rues comme dans les salons ${ }^{17}$. Le Courrier du Canada sacrifiait même les nouvelles locales pour commenter les derniers bulletins transmis par le télégraphe intercontinental ${ }^{18}$. Le 18 août quatre à cinq cents Canadiens français se réunissaient chez le Consul de France, M. Gauthier. Ils voulaient créer un Comité pour réunir des secours destinés aux blessés français. A la fin de la réunion l'avocat Amyot réclama l'exécution du Chant national de la France. De tous côtés on cria "La Marseillaise, la Marseillaise". Les assistants se rangèrent en cortège et s'ébranlèrent aux premiers mots de la Marseillaise; "Allons, enfants de la patrie...". Le cortège prit la rue Buade, descendit la rue de la Fabrique, dégringola la côte d'Abraham jusqu'à la Basse-Ville. De minute en minute le flot se grossissait de nouveaux manifestants "personnes de tout rang et de tout âge, aristocrates et démocrates, vieillards et jeunes gens... On marchait quatre par quatre, bras dessus bras dessous". ${ }^{19}$

Le 28 août, 2,000 personnes se réunissaient à la salle JacquesCartier. Elles exprimaient "leurs plus sincères sympathies pour la
15. 10 aout.
16. 20 Août.
17. Courrier du Canada, 24 aout.
18. Evenement, 19 aout 1870.
19. Courrier du Canada, 29 aodt. 
France dans la glorieuse lutte qu'elle soutient contre la Prusse" et faisaient des vœux pour son complet succès. Elles affirmaient "que c'était un devoir pour tous de contribuer à la souscription en faveur des blessés français". On décidait que des membres du C,omité de souscription visiteraient les citoyens à domicile pour recevoir les cotisations. A la fin de la séance un chanteur de la paroisse St-Roch, entonnait l'hymne national de la France qui fut répété en chœur. L'Assemblée se dispersait aux cris de "Vive la France"19.

Cette explosion de sympathie envers la France ne se limita pas à Québec. On la retrouve à Montréal ${ }^{20}$, à Trois-Rivières, à StHyacinthe, à Ottawa. ${ }^{21}$ A St-Hyacinthe quelques jeunes gens se forment en cortège à 7 heures du soir et entonnent "La Marseillaise" avec un entrain que le patriotisme parisien n'aurait pas désavoué. Ils sont bientôt rejoints par un grand nombre de personnes de tout âge. Parvenus sur la place du Marché, ils sont près de $200^{22}$. Dans les plus petits villages des campagnes, au dire du Courrier du Canada, les bureaux de poste étaient envahis à l'arrivée de chaque courrier par une foule pressée d'avoir des nouvelles du théâtre de la guerre ${ }^{23}$. Mais il semble bien que Québec se signala par sa ferveur française. L'Opinion publique de Montréal lui rendait hommage en ces termes: "La vieille cité de Champlain est toujours la ville française par excellence... Lorsque Québec n'aura plus de patriotisme, les eaux du Saint-Laurent auront remonté vers leur source ${ }^{24}$."

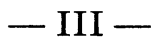

Le 2 septembre 1870 le Courrier du Canada prophétisait: "Le jour de la revanche française arrive". Le même jour la Revue Canadienne publiait un poème d'Eustache Prud'homme, plein d'optimisme:

19. Courrier du Canada, 29 août.

20. Evenement, 24 aout.

21. Evenement, 31 août.

22. Courrier du Canada, 27 août.

23. Courrier du Canada, 24 aodt.

24. Cité par le Courrier du Canada, 2 sept. Dans sa livraison d'août 1870 (Vol. 7: 616), la Reoue Canadienne notait ce trait amusant: "Le conflit franco-prussien a tellement préoccupé les esprits que la proclamation du dogme de l'infaillibilité pontificale a passé presque inaperçu". 
O France, on a voulu démembrer ton empire...

Il est enfin venu ce jour de la vengeance

Ce jour où les Prussiens, jaloux de ta puissance,

Vont joncher de leurs os tes coteaux dévastés.

Ah! sais-tu que bien loin, par delà l'Atlantique,

Il est un peuple ardent, jeune et patriotique

Qui porte sa noblesse écrite sur le front?

Sais-tu que nos aïeux sont nés sur les rivages

Et qu'après trois cents ans de luttes et d'orages

Nous aimons à dire ton nom?

Oui tu vaincras malgré les armes qu'on t'oppose ${ }^{25}$.

Ce même jour pourtant Napoléon III capitulait à Sedan avec une armée de 100,000 hommes. Le 4 septembre Paris proclamait la République.

Québec souffrit profondément de la capitulation de Sedan et de la Révolution parisienne. "Les habitants sont consternés et comme foudroyés dans leur plus chère affection, note l'Evénement ${ }^{26} .$. Nous n'avons jamais vu pareil recueillement, pareille douleur. La France vaincue recevra des hommages plus retentissants, jamais un témoignage plus sincère d'affection. Notre propre patrie écrasée, notre propre sol dévasté, n'auraient pas causé à nos âmes une souffrance plus cruelle, arraché à nos poitrines un sanglot plus déchirant. Le peuple canadien tient encore à la France par toutes les fibres du cœur."

Par ailleurs la République épouvante: elle rappelle les jours sanglants de la grande Révolution et les émeutes de 1848. En vain l'Événement plaide la cause du nouveau gouvernement parisien. "Il n'y pas dans le cabinet un seul révolutionnaire au sens mauvais du mot... On est frappé du caractère de modération et de sagesse du gouvernement provisoire... Trochu, c'est un mélange de Cavaignac et de Lamoricière... Jules Favre est un orateur accompli... Gambetta est l'homme le plus éminent du cabinet, orateur hors ligne, auquel Emile Ollivier ne répondait qu'en tremblant et dont la parole vigoureuse et éloquente a révélé de suite la supériorité d'esprit ${ }^{27}$." Il est le seul journal de Québec à approuver la République française.

25. Revue Canadienne, VII: 670.

26. Numéro du 5 sept.

27. 9 septembre. 
Le Courrier du Canada se lamente à grands cris: "La France est livrée aux mains d'une poignée de socialistes et d'impies... Jules Simon est un matérialiste... Rochefort a exprimé dernièrement son regret d'avoir reçu le baptême... Gambetta a prostitué son talent à la religion de $89 .$. Picard a pris, entre autres engagements, celui de démolir la Papauté" (12 septembre). Le Journal de Québec donne encore davantage dans la violence: "Les républicains qui gouvernent la France sont les rebuts de la population; ...des partageux qui veulent avoir leur part du bien des riches;... Paris aimera mieux se voir sous la domination des Prussiens qu'à la merci des amis de Rochefort et des Disciples de Proud'hon".

Naturellement le thème principal des commentaires de la presse québécoise tourne autour de la question des responsabilités: sur qui doit retomber la responsabilité d'une telle catastrophe? "En 1815 , note avec justesse le Canadien, après 20 ans de guerre, le grand Napoléon devait naturellement et noblement succomber contre toute l'Europe coalisée. Mais aujourd'hui cette même France, plus forte et plus puissante que jamais, est venue se briser en quelques jours contre les gros bataillons d'une seule puissance, la Prusse, son ancien adversaire qu'elle a tant de fois vaincu." (5 septembre).

Dans cette recherche des responsabilités les journaux québécois divergent d'opinion. Trois thèses différentes sont avancées. Le Journal de Québec et le Canadien tiennent pour la trahison. Napoléon III a été trahi par ses ennemis républicains qui l'ont empêché de préparer la guerre. "En fait, affirme le Canadien, le 12 septembre, le règne entier de Napoléon III a été une ère de paix, d'ordre, de prospérité et de progrès matériel. Sous son gouvernement la France a repris sa place à la tête de la civilisation moderne; sa capitale a été florissante; ses manufactures ont pris un développement prodigieux; elle est devenue la seconde puissance commerciale et maritime du monde". Le 7 septembre le Journal de Québec protestait contre les Parisiens qui détruisent les statues de l'Empereur, "de l'homme pratique qui avait fait la France riche et puissante, et qui l'eût rendue comme auparavant victorieuse, sans ces apôtres de la paix à tout prix et du désarmement", c'est-à-dire sans "l'utopiste Jules Favre" et "la canaille" qui l'entoure.

28. Canadien, 5 et 12 septembre. Journal de Québec, 5-7 septembre. 
Organe du libéralisme et favorable à la proclamation de la République française, l'Événement ne pouvait que soutenir une thèse inverse. A qui la faute? Mais à Napoléon III lui-même. Il s'est emparé de la France à la suite d'une "réaction honteuse". "Pendant 18 ans Napoléon et son entourage n'ont travaillé qu'a affaiblir la France, afin de se maintenir... Le régime impérial a émasculé la France... Il a usé les forces de la nation et installé partout la corruption... Il éblouissait de loin,... C'était une terne figure, qui rappelle à s'y méprendre un Empereur du Bas-Empire ${ }^{29}$."

Quant au Courrier du Canada, sa réponse était métaphysique. La défaite française, c'était le dọigt de la Providence. L'idée est largement exposée dans l'Editorial du 7 septembre. "La clef de sa défaite se trouve dans cette phrase: quos vult perdere, Deus prius dementat. Dieu commence par aveugler ceux qu'il veut perdre. La Providence a voulu punir du même coup le chef et ses sujets: le chef pour avoir oublié qu'il était le serviteur de l'Église ${ }^{30}$; les sujets pour avoir élevé des statues à Voltaire dans le siècle qui a vu la définition du dogme de l'Immaculée-Conception. Dieu a parlé à l'empereur Napoléon III et à la France par la bouche des mitrailleuses prussiennes."

Malgré la sévérité des jugements, - on n'est sévère que pour ceux que l'on aime - la sympathie québécoise restait entière pour la France. Le 8 septembre était publiée la première liste de souscriptions pour les blessés français. Elle s'élevait à 1310 dollars. Parmi les souscripteurs on relevait les noms suivants:

L'Honorable I. Thibaudeau............. 100.

L'Honorable Juge Taschereau.......... 50.

L'Honorable P.-J. Chauveau ........... 25.

L'Honorable Cauchon.............. 25.

L'Honorable Chaussegros de Léry ......... 25.

Les membres du club Jacques-Cartier...... 25.

Les Frères des Ecoles Chrétiennes....... $20^{31}$.

29. Voir surtout les numéros de l'Evénement du 7 et 8 septembre.

30. Dans son numéro du 5 septembre le Courrier du Canada rapprochait Sedan de Waterloo et Napoléon III de Napoléon I. "Comme son oncle Napoléon III a rendu des services à l'Église au début de son règne, mais, comme lui aussi, il a tourné le dos à l'Église en assistant, impassible et presque complice, au démembrement du territoire pontifical au profit de l'Italie".

31. Evenement, 8 sept. 
Quelques jours plus tard devaient souscrire l'Archevêque de Québec et le Séminaire ${ }^{32}$. Le 19 septembre le Consul Général de Québec remerciait les Québécois de leur générosité ${ }^{33}$. Le 27 septembre Jules Favre, ministre des Affaires Etrangères, remerciait à son tour ${ }^{34}$. "Si le souvenir de leur ancienne mère patrie est resté vivant dans le cour des Canadiens-français, la France elle aussi n'a pas perdu leur souvenir; elle est toujours fidèle à sa vieille amitié pour les habitants de cette terre autrefois française."

Faut-il d'ailleurs désespérer de l'issue de la guerre? Non pas, répondent unanimement les journaux de Québec en ce tragique mois de septembre. Et tous d'alleguer les raisons d'espérer ${ }^{35}$. La Prusse est haletante et épuisée. L'Europe ne laissera pas démembrer la France. Si la Prusse demande plus que les frais de la guerre, il se formera contre elle une coalition plus formidable que celle qui s'était formée autrefois contre la France. Le peuple de Berlin verra à son tour l'Gtranger à ses portes. Au surplus en France se lèvent de nouvelles armées pour repousser l'envahísseur. Bazaine résiste toujours à Metz. Paris sera le tombeau des Prussiens.

Le 9 septembre, Le Canadien écrivait: "L'existence de la France est nécessaire à la civilisation et à la religion. Dieu ne l'abandonnera pas tout à fait. Espérons en elle."

$$
\text { - IV - }
$$

De fait un moment la victoire parut hésiter. L'énergie de Gambetta suscitait de nouvelles armées. Paris assiégée refusait de se rendre. Les troupes françaises remportaient quelques succès. Vains efforts, hélas! Le 28 janvier 1871 Paris capitulait. Malgré l'héroïsme des six derniers mois la guerre franco-allemande se terminait en désastre.

De nouveau la presse québécoise posa le problème des responsabilités et chercha le bouc émissaire. Un duel fort amusant et

32. Evenement, 30 sept.

33. Evenement, 19 sept.

34. Evenement, 27 sept.

35. Il m'est impossible de citer toutes mes références. C'est presque tous les numéros de septembre qu'il faudrait énumérer, et dans chaque journal. Je ne fais que résumer les principaux arguments. 
parfois violent s'engagea à ce sujet entre deux journaux de Québec: le Courrier du Canada et l'Événement. L'épisode mérite d'être conté. $\mathrm{Au}$ Courrier du Canada, écrivait Basile Routhier. Jeune encore - il n'avait que 31 ans - il avait adopté toutes les idées de Louis Veuillot, même ses allures de Grand Juge ${ }^{36}$. A ses yeux le fait est clair. "La France est châtiée par Dieu et elle l'a bien mérité ${ }^{37}$." Il ne faut pas dire: La France n'était pas prête à faire la guerre, elle n'avait pas assez de rógiments, pas assez d'artillerie, pas assez de discipline. C'est la Providence qui a voulu que la France ne fut pas prête. La Prusse n'a été entre ses mains qu'un instrument aveugle et sans mérite, comme le fut autrefois Nabuchodonosor. Ce n'est pas la première fois que Dieu se venge. La France était sérieuse et grande dans la première moitié du XVIIe siècle. Mais ensuite elle s'abandonna entièrement au rire grivois de Molière et de La Fontaine: alors Dieu l'accabla de revers. Depuis 1789 et la grande révolution satanique, la France a choisi pour mère une marâtre, qui s'appelle La Liberté, et cette infâme l'a pervertie. Voltaire et Victor Hugo ont remplacé Dieu. Les Français se sont abandonnés à la folie du plaisir. Paris est devenu la capitale de Satan en ce monde. Bien plus encore: Napoléon III a livré le pape aux sicaires de la Révolution. Il a fermé la bouche aux écrivains catholiques, il a supprimé la conférence saint Vincent de Paul, mais laisse toute liberté aux journaux impies et aux théâtres immoraux. Dieu s'est vengé. La punition de la France s'est d'abord manifestée par la décadence de ses mœurs et de sa civilisation. Ses philosophes sont des plagiaires. Ses orateurs sont des phraseurs. Ses poètes, y compris Victor Hugo, sont des rêveurs excentriques, ses hommes politiques trahissent l'ignorance la plus grossière. Maintenant Paris va être foulé aux pieds par Attila. Le 28 octobre, l'Editorial du Courrier du Canada se permettait même de souhaiter la destruction de la capitale française: "Nous dirons que la prise de Paris n'est pas chose impossible. et que nous croyons aux prédictions qui disent que Paris sera détruit. Nous y

36. D'après la Revue Canadienne, 1871, VIII: 952. "Plusieurs des admirateurs de Mr Routhier ont porté l'enthousiasme jusqu'à lui décerner le titre de Louis Veuillot canadien".

37. Je n'ai pas la place de citer intégralement Routhier. Je résume seulement ses articles. Il faut lire surtout les articles des 23 et 30 septembre, 10 octobre, 2 novembre. 
croyons à cette prédiction, parce que la capitale de la France a mérité par sa dépravation le châtiment de Sodome... c'est Paris qui doit solder le compte de la justice divine ${ }^{38}$."

Après Routhier d'autres allaient reprendre cette thèse du châtiment divin infligé à la France pour ses péchés, notamment l'abbé Raymond dans un grand discours prononcé devant l'Union Catholique de Saint-Hyacinthe, le 8 décembre 1870. L'orateur commençait par rappeler que "la guerre est un châtiment de la justice divine ${ }^{39}$."

L'histoire le prouvait: Contre Juda le Seigneur appelle les armes terribles des rois d'Assyrie; quand Balthazar a mis le comble à ses vices par une insigne profanation, le glaive de Cyrus lui ôte la vie. L'invasion des Barbares a eu lieu à l'époque où le paganisme, vaincu dans les idées, triomphait des mœurs chrétiennes. Le terrible glaive de Mahomet et de ses successeurs n'a frappé que des nations en proie à l'hérésie ou aux mœurs dissolues. La guerre de Trente Ans, qui a amené de si affreux désastres en Allemagne, a été l'effet et la punition du schisme de Luther.

Et l'orateur continuait: "Jadis la France était chrétienne: "Un grand pape avait dit d'elle: la France est le plus beau royaume après celui du ciel. Son bras était l'instrument des actes de Dieu. Gesta Dei per Francos. Elle avait constitué l'indépendance de la Papauté; dans son sein était éclose la noble fleur de la chevalerie; son cœur avait conçu l'hérołssme des croisades...

Mais depuis le XVIIIe siècle la France n'est plus chrétienne: Avec Voltaire, de toutes parts, on entendit... le cri prononcé par le peuple déicide: nous ne voulons pas que le Christ règne sur nous. Dieu fut banni de la société.

Alors éclata cette épouvantable catastrophe qui s'appelle la Révolution française. En ce temps-là, la souveraineté fut donnée à la guillotine. L'humble hache, après avoir coupé la tête du Monarque de la France, abattit celle des princes, des princesses, des nobles, des évêques, des prêtres, des religieux et religieuses, de tout ce qu'il y avait de chrétien, de loyal, d'honnête chez les femmes comme chez les hommes.

Après vint l'Empire. Quei que fût l'éclat de ses conquêtes, c'était

38. L'Editorial n'est pas signé.

39. Discours reproduit dans la Revue Canadienne de juin 1871, VIII: 27-56. 
le joug d'un despotisme avilissant, produisant dans la nation une vraie dégradation sociale... La France était alors un peuple de soldats et de valets...

Les anciens princes revinrent... Mais l'esprit irréligieux ramena la Révolution qui les renversa... 18 ans plus tard la Révolution proclama la République, qui, attaquée bientôt par l'anarchie, n'en triompha qu'en inondant de sang les rues de Paris...

C'est alors que reparut le nom de Napoléon... La France se fit encore valet.

L'immoralité atteignit son comble, immoralité qui "dans une grande partie de la population ne rougit de rien et cherche à étendre son infection de plus en plus. La capitale de la France, centre de ces iniquités et de ces immondices, ne nous apparaît plus que comme une terre souillée, ainsi que celle de Babylone ou de Sedan et, comme telle, appelant les vengeances du Ciel."

De telles violences verbales dépassaient évidemment la mesure. Dans l'Evénement, Hector Fabre se permit de protester contre l'usage abusif de l'argument providentiel substitué aux causes secondes: "L'histoire n'est pas un recueil de pénitences à l'usage des peuples... la défaite de la France s'explique par des causes humaines". Il se permit également deux remarques ironiques. Dieu punit la France par la Prusse, mais alors il récompense la Prusse. Or Louis Veuillot n'a-t-il pas dit que "la Prusse était le péché mortel de l'Europe" ?. Par ailleurs, si Paris est châtié pour ses péchés, il faut en dire autant du Pape. Les Italiens ne venaient-ils pas de s'emparer de Rome? ${ }^{40}$

Naturellement Routhier bondit sous l'attaque. Dieu se sert de la Prusse; il ne la récompense pas. "Bientôt viendra le châtiment de la Prusse. Dieu se rira d'elle et de son immense orgueil, comme il a ri de la France, et il la cassera comme un verre: Irridebit et conquassabit. D'autre part il est scandaleux d'assimiler Rome et Paris. Rome c'est le juste qui souffre, Paris c'est le pécheur qui est puni. La France a trahi ses devoirs: elle a abandonné l'Eglise. A Rome, il y a un pontife vénéré qui n'a pas commis de faute. Non, il n'y a pas ici expiation, mais épreuve".

40. Toute la polémique est résumée dans un article de Routhier du Courrier du Canada (12 décembre). 
Fabre réplique à Routhier par une épithète malveillante: il n'est que "la tremblante image" de L. Veuillot", puis il l'accuse d'hypocrisie et le compare au Basile de Beaumarchais ${ }^{42}$.

Routhier recourt lui aussi à l'injure. Fabre est un Figaro "type du courtisan, de l'intrigant et de l'homme sans principes". Il est aussi Coquelet "création de Louis Veuillot qui personnifie la libre-pensée 43 ". Le 17 janvier Routhier était encore plus violent: Fabre ne sait même pas écrire le français. C'est le plus vil, le plus lâche, le plus éhonté des menteurs.

Tout se termina par des chansons. Le 20 janvier 1871 le Courrier du Canada publiait une manière de pièce de théâtre. La scène se passait à Québec. Le théâtre représentait une rue droite qui se terminait par un escalier tortueux. Au haut de l'escalier une boutique de mauvaise apparence avec cette enseigne: l'EVENEMENT.

Sur la porte entr'ouverte apparaît Figaro-Coquelet. Il chante:

Du Catéchisme et de l'Histoire

$J_{\Theta}$ ne sais pas un traître mot.

Mais je m'en moque et m'en fais gloire.

Apprendre est le métier d'un sot.

Tout est instable sur la terre

Rien ne doit être respecté

Se moquer est la grande affaire

Et croire est une absurdité

D'après le Courrier du Canada (27 janvier) le morceau aurait fait le tour de la presse.

De toutes ces discussions, la France sortait assez malmenée. Elle n'était pas la seule. La défaite française semble avoir eu pour contre-coup de déchaîner dans la presse québécoise un violent accès de xénophobie. Ulcérés dans leur amour-propre et dans leur loyalisme sentimental à l'égard de la mère-patrie, les québécois semblent dire: La France ne vaut rien. Bien. Mais les autres nations valent moins encore.

41. Courrier du Canada, (21 décembre).

42. Courrier du Canada, 4 janvier 1871.

43. Courrier du Canada, 4 janvier 1871. 
La Prusse est quotidiennement injuriée. Ses soldats se conduisent comme des brutes: ils brâlent les visages, fusillent de sang-froid les francs-tireurs, violent puis assassinent les femmes ${ }^{44}$. Il est faux de parler de la supériorité des Allemands sur les Français. L'Allemand est un automate; il rit généralement un quart d'heure après qu'il a entendu une plaisanterie. Il n'a aucune politesse, mais de la vanité de la plus singulière espèce. Les Allemands accusent les Français de légèreté dans leurs mœurs. "Allez à l'opéra de Berlin et vous m'en direz des nouvelles" ${ }^{45}$. En tout cas ils ne respectent rien et sont tous plus ou moins imbus des idées du vieux Blücher, ce pillard émérite qui, se trouvant à Londres après les guerres de l'Empire, ne put s'empêcher de dire, en contemplant l'immensité de Londres, du haut de St-Paul: "quelle ville pour un pillage" 46 . Bismark revendique l'Alsace et la Lorraine: ce sont terres françaíses sur lesquelles l'Allemagne n'a que le droit de la force ${ }^{47}$.

De l'Italie il en est peu question, mais toujours avec le dernier mépris. C'est la nation qui ose s'attaquer au Pape, et encore sans vouloir courir le moindre risque. Elle a attendu le départ des divisions françaises de Rome pour attaquer la faible armée pontificale ${ }^{48}$.

La Russie tsariste est la complice de l'Allemagne. Pour prix de sa neutralité, Bismark lui a promis l'Orient, Constantinople, les Indes même ${ }^{49}$. Routhier prophétise, dans le Courrier du Canada du 10 octobre: "Si l'Europe ne revient pas à Dieu, l'Europe sera livrée au dieu qu'elle s'est choisi, la matière, et la matière l'écrasera. Après l'heure de la Prusse sonnera l'heure de la Russie et le monde sera perdu."

Mais c'est surtout l'Angleterre qui a droit aux sarcasmes les plus désobligeants. Elle doit à la France d'avoir gagné la guerre de Crimée: elle solde sa dette par un refus d'intervenir dans la guerre franco-allemande. Ainsi le veulent les "préférences dynastiques de
44. Evenement, 19 octobre.
45. Evénement 18 octobre.
46. Journal de Quebec, 8 oct.
47. Canadien, 14 oct. 1870.
48. Evénement, 6 septembre.
49. Evenement, 15 nov. 
sa maniaque souveraine" ${ }^{55}$. Thomas Carlyle, l'historien anglais, s'est déshonoré en écrivant au Times: "Après avoir souffert, pendant quatre siècles du fâcheux voisinage de la France, l'Allemagne a enfin le grand bonheur de voir la France tout à fait à bas... Après de telles victoires et une telle provocation, la demande des Allemands sur l'Alsace-Lorraine semble raisonnable, juste et modérée ${ }^{51}$."

Les journaux anglais du Canada ne cessent de broder sur le thème des humiliations de la France. Que ne parlent-ils des humiliations de l'Angleterre? La France se défend. L'Angleterre préfère la mort au combat. Elle a reculé dans l'affaire du Danemark. Elle a abandonné la Mer Noire aux Russes et le Luxembourg à la Prusse. Elle abandonne maintenant le Canada aux États-Unis: elle retire du Canada toutes ses troupes. Elle n'y laisse ni un soldat, ni une cartouche, ni un canon. Il y a de quoi faire oublier le déshonneur de Sedan. Gladstone n'est ni Pitt ni Palmerston: il n'est bon qu'à faire des discours et à pérorer à perte de vue sur des questions d'économie politique. L'Angleterre, en cédant, cédant toujours, est devenue une puissance de second, sinon de troisième ordre. Elle préfère le côté des plus forts, et elle sacrifie tout dans la crainte de tirer un coup de canon ${ }^{52}$.

L'avenir réservera des surprises amères à l'Angleterre. L'Europe sera dominée par l'Allemagne. Les tsars de Russie auront l'Asie, sans excepter les Indes. Les États-Unis prendront le Canada. Toujours avide de prophéties sensationnelles, le Courrier $d u$ Canada déclare le 23 septembre:

La plupart des Anglais qui applaudissent aujourd'hui aux succès de la Prusse vivront assez pour voir la déchéance de l'Angleterre; et le châtiment n'aura pas été volé.

Faut-il conclure? Je laisse la parole à Wilfrid Laurier. Dans un discours prononcé à Paris le 2 août 1897 il disait:

"Séparés de la France, nous avons toujours suivi sa carrière avec un intérêt passionné, prenant notre part de ses gloires et de ses triomphes, de ses joies et de ses deuils, de ses deuils surtout. Hélas!

50. Evenement, 15 nov.

51. Journal de Quebec, 17 décembre.

52. Canadien, 21 déc. 1870. Courrier du Canada, 3 octobre. 
Jamais nous ne sûmes peut-être à quel point elle nous était chère que le jour où elle fut malheureuse. Oui, ce jour-là, si vous avez souffert, j'ose le dire, nous avons souffert autant que vous. ${ }^{53}$ "'

Alfred Rambaud, Professeur d'Histoire Moderne d l'Université Laval.

53. Cité par Siegfried, Le Canada, Les deux races, 141. 